\title{
Advantages of dental implant
}

\author{
Seong Hyeok CHOI, Jae Kyung RYU, Kyung Sook HWANG* \\ Dept. of Dent. Tech., Shin Han Univ., Korea
}

The main discourse: 1 . Appearance is very similar to natural teeth. If you don't tell yourself, others don't know because you can also see the shape and color of adjacent teeth. Thus, it is in harmony with the other teeth of the oral cavity.

2. For teeth where the occlusion does not match, an artificial crown prosthesis can be used to create a harmonious occlusion between the maxilla and the mandible. Therefore, it can increase the efficiency of writing and can be aesthetic.

3. This prevents problems in the oral cavity after tooth loss. Prevents pathological movements of adjacent teeth or deformation of the face in advance. In particular, if teeth are left in a missing state for a long time, the mating teeth may be seated and adjacent teeth may be severely tilted, resulting in insufficient space for the crown prosthesis.

4. If the missing part is left as it is, the gums may become weak due to insufficient stimulation of the gums by food. Therefore, it is possible to prevent the loss of alveolar bone with age or the gradual loss of bone that may occur in the missing area by repeating the stimulation like a natural tooth after implant placement. For reference, it takes a lot of time to fill the root part extracted from periodontal disease with alveolar bone. However, alveolar bone formation of alveolar bone is relatively fast in tooth extraction due to tooth decay or in younger age groups.

5. Implant prostheses have a very long lifespan. Conservatively, it is generally recommended to replace the crown every 10 years.

6. Some people have no teeth due to a congenital lack of permanent teeth, and they must get an implant. Sometimes used as a replacement for permanent teeth, but never recommended. Because the baby teeth themselves are much smaller than permanent teeth and have short roots, the baby teeth do not last long due to lack of support. Therefore, in this case, it should be replaced with an implant when entering adolescence.

7. Because it can maintain chewing power for a long time, it reduces the burden on the stomach on digestive function, and is essential for maintaining body health because it allows you to eat and absorb healthy nutrients. Chewing strength is important to keep your body healthy for a long time. There will be many more.

Keywords: Advantages of implant, histological, anatomical implant

Acknowledgement: This study is supported by Uniance's Central Research Institute.

Copyright (C) 2021. Korean Academy of Preventive Dentistry. All rights reserved.

This is an Open Access article distributed under the terms of the Creative Commons Attribution Non-Commercial License (http://creativecommons.org/licenses/ by-nc/4.0) which permits unrestricted non-commercial use, distribution, and reproduction in any medium, provided the original work is properly cited. 